\title{
Living Cationic Copolymerization of Isobutyl Vinyl Ether with p-Methoxystyrene by Hydrogen Iodide/Iodine Initiating System
}

\author{
Mitsuo SAwamoto, Takehiko Ohtoyo, Toshinobu HigashimURA, \\ Karl-Heinz GÜHRS, * and Günther HEUBLEIN* \\ Department of Polymer Chemistry, Faculty of Engineering, \\ Kyoto University, Kyoto 606, Japan \\ * Department of Chemistry, Friedrich-Schiller-University, \\ DDR-6900 Jena, German Democratic Republic
}

(Received February 7, 1985)

\begin{abstract}
Living cationic copolymerization of isobutyl vinyl ether with $p$-methoxystyrene was effected by an equimolar mixture of hydrogen iodide and iodine $\left(\mathrm{HI} / \mathrm{I}_{2}\right.$ initiator $)$ at $-15^{\circ} \mathrm{C}$ in nonpolar solvents (carbon tetrachloride and toluene). Isobutyl vinyl ether reacted faster than $p$ methoxystyrene. The products were random copolymers with a nearly monodisperse molecular weight distribution (MWD) $\left(M_{w} / M_{n}=1.1-1.2\right)$. Their number-average molecular weight $\left(M_{n}\right)$ increased in proportion to total monomer conversion. The iodine-initiated copolymerization under similar conditions led to long-lived (but not perfectly living) copolymers with a narrow MWD $\left(M_{w} / M_{n}=1.2-1.3\right)$. In contrast, boron trifluoride etherate induced a conventional transferdominant copolymerization. The $M_{n}$ of the copolymers obtained with the $\mathrm{HI} / \mathrm{I}_{2}$ initiator was almost independent of the monomer feed ratio, but in the $\mathrm{BF}_{3} \mathrm{OEt}_{2}$-initiated non-living process, the polymer molecular weight decreased sharply with increasing isobutyl vinyl ether content in the feed. These findings show the living copolymerization by $\mathrm{HI} / \mathrm{I}_{2}$ to be free from the cross-transfer usually involved in the conventional cationic copolymerization of vinyl monomers.
\end{abstract}

KEY WORDS Cationic Copolymerization / Living Polymer / Monodisperse Polymer / Cross-Transfer / Isobutyl Vinyl Ether / $p$-Methoxystyrene / Hydrogen Iodide / Iodine / Narrow Molecular Weight Distribution /

In most cationic copolymerizations between vinyl compounds, the molecular weight of the product copolymers is much less than those of the respective homopolymers. ${ }^{1}$ This molecular weight depression of copolymers is attributed to cross-transfer ${ }^{2,3}$ that occurs particularly when the monomers to be copolymerized differ considerably in reactivity and structure (e.g., isobutene $v s$. styrene ${ }^{2}$ and vinyl ethers $v s$. styrenes ${ }^{4}$ ). Accordingly, the synthesis of (random) copolymers with controlled molecular weight and narrow molecular weight distribution (MWD) has been difficult in cationic vinyl copolymerization.

Recently we have found nearly perfect "living" polymerizations of vinyl ethers ${ }^{5,6}$ and related monomers ${ }^{7,8}$ initiated by a mixture of hydrogen iodide and iodine $\left(\mathrm{HI} / \mathrm{I}_{2}\right.$ initiator). In view of the absence of chain transfer (and termination) in living polymerization, it is of interest to apply the $\mathrm{HI} / \mathrm{I}_{2}$ initiating system to cationic "copolymerizations" that may involve frequent cross-transfer reaction(s) when initiated by conventional acid initiators.

Thus, in this study, we copolymerized, using the $\mathrm{HI} / \mathrm{I}_{2}$ initiator, isobutyl vinyl ether (IBVE) and $p$-methoxystyrene (pMOS) which differ in structure and reactivity and undergo (cross)transfer dominant cationic copolymerization under usual conditions. ${ }^{1,4}$ Our object was to 
prevent cross-transfer and other chainbreaking reactions so as to control polymer molecular weight and MWD and thus be able to synthesize "living random" IBVE-pMOS copolymers with a monodisperse MWD, hitherto not yet prepared.

\section{EXPERIMENTAL}

\section{Materials}

IBVE (Tokyo Kasei) was washed with aqueous sodium hydroxide solution and then water, dried over potassium hydroxide pellets, and distilled twice before use over calcium hydride and then sodium metal. pMOS was prepared from $p$-methoxyacetophenone by the literature $\operatorname{method}^{9}$ and purified by double distillation over calcium hydride under reduced pressure. The two monomers were of gas chromatographic purity exceeding $99.8 \%$.

Hydrogen iodide was obtained as an $n$ hexane solution $(c a .1 \mathrm{M})$ by dehydration of a commercial $57 \%$ aqueous solution as reported. ${ }^{5,6}$ Iodine was sublimed over potassium iodide. Boron trifluoride etherate $\left(\mathrm{BF}_{3} \mathrm{OEt}_{2}\right)$ was purified by vacuum distillation. These initiators were stored under dry nitrogen in ampules in the dark. Solvents (carbon tetrachloride, toluene, and $n$-hexane) and tetrahydronaphthalene (internal standard for gas chromatography) were washed successively with sulfuric acid, aqueous sodium hydroxide, and water, and doubly distilled over calcium hydride before use.

\section{Procedures}

The copolymerization of IBVE with pMOS was carried out under dry nitrogen in a baked glass tube equipped with a three-way stopcock. The reaction was initiated by adding an initiator solution to a monomer solution via dry syringe and teminated with prechilled ammoniacal methanol. When $\mathrm{HI} / \mathrm{I}_{2}$ initiator was employed, hydrogen iodide and iodine solutions were added successively in this order. The concentration of hydrogen iodide stock solutions was determined by titration as reported. ${ }^{6}$ Conversions of the two monomers were measured by gas chromatography on a Shimadzu temperature-programmed gas chromatograph GC-6A with tetrahydronaphthalene as internal standard (ca. $5 \mathrm{vol} \%$ ): column, PEG 6000 (Shimadzu), $3 \mathrm{~mm}$ i.d. $\times 2 \mathrm{~m}$; carrier gas, hydrogen, $1.0-1.5 \mathrm{~kg} \mathrm{~cm}^{-2}$; the column temperature was raised from 60 (for IBVE) to $180^{\circ} \mathrm{C}$ (for pMOS and tetrahydronaphthalene) at the rate of $10 \mathrm{deg} \mathrm{min}^{-1}$.

The quenched reaction mixture was washed with aqueous sodium thiosulfate solution ( $\mathrm{ca}$. $10 \% \mathrm{w} / \mathrm{v}$ ) and then water, evaporated to dryness under reduced pressure below $40^{\circ} \mathrm{C}$, and vacuum-dried to give the product polymers. For runs with $\mathrm{BF}_{3} \mathrm{OEt}_{2}$, treatment with sodium thiosulfate was omitted. The MWD of the copolymers was measured by size exclusion chromatography (SEC) in chloroform on a JASCO Trirotar-II chromatograph equipped with three polystyrene gel columns (Shodex A802, A-804, and A-806, $8.0 \mathrm{~mm}$ i.d. $\times 500 \mathrm{~mm}$ each) and refractive index (RI)/ultraviolet (UV) dual detectors. The number-average molecular weight $\left(M_{n}\right)$ and polydispersity ratio $\left(M_{w} / M_{n}\right)$ of the polymers were calculated from SEC eluograms on the basis of a polystyrene calibration. ${ }^{1} \mathrm{H}$ NMR spectra were recorded in $\mathrm{CDCl}_{3}$ at $30^{\circ} \mathrm{C}$ on $\mathrm{JEOL}$ FX 90Q spectrometer.

\section{RESULTS AND DISCUSSION}

\section{Living IBVE-pMOS Copolymerization by $\mathrm{HI} / \mathrm{I}_{2}$ Initiator}

Our previous studies showed that nonpolar solvents are best suited for the living polymerization of IBVE by $\mathrm{HI} / \mathrm{I}_{2}$ initiator $^{5}$ and the formation of long-lived polymers from pMOS by iodine. ${ }^{10,11}$ For this reason, IBVE and pMOS were copolymerized (IBVE/pMOS = $1 / 3$ in feed) by an equimolar mixture of hydrogen iodide and iodine at $-15^{\circ} \mathrm{C}$ in carbon tetrachloride, a good solvent for the homopolymers of the two monomers. Iodine was 


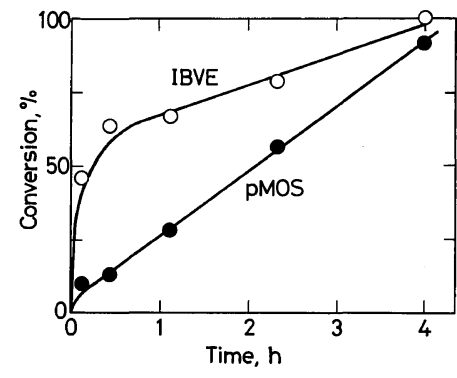

Figure 1. Time-conversion curves for IBVE-pMOS copolymerization by $\mathrm{HI} / \mathrm{I}_{2}$ at $-15^{\circ} \mathrm{C}$ in $\mathrm{CCl}_{4}$ : $[\mathrm{IBVE}]_{0}=$ $0.19 \mathrm{M} ;[\mathrm{pMOS}]_{0}=0.57 \mathrm{M}(\mathrm{IBVE} / \mathrm{pMOS}=1 / 3) ;[\mathrm{HI}]_{0}=$ $\left[\mathrm{I}_{2}\right]_{0}=10 \mathrm{mM}$.
(A) $\mathrm{HI} / \mathrm{I}_{2}$

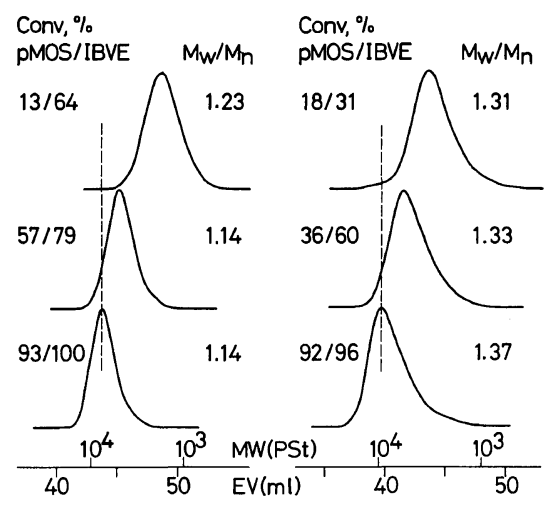

Figure 2. MWD of IBVE-pMOS copolymers obtained with $\mathrm{HI} / \mathrm{I}_{2}$ (A) and iodine (B) at $-15^{\circ} \mathrm{C}$ in $\mathrm{CCl}_{4}$ : $[\mathrm{IBVE}]_{0}=0.19 \mathrm{M} ; \quad[\mathrm{pMOS}]_{0}=0.57 \mathrm{M} \quad(\mathrm{IBVE} / \mathrm{pMOS}=$ $1 / 3) ; \quad$ (A) $[\mathrm{HI}]_{0}=\left[\mathrm{I}_{2}\right]_{0}=10 \mathrm{mM} ;$ (B) $\left[\mathrm{I}_{2}\right]_{0}=20 \mathrm{mM}$. Conversion and $M_{w} / M_{n}$ as indicated.

also used as an initiator under the same conditions. Figures $1-3$ summarize the results.

With both initiators, IBVE and pMOS were polymerized without an apparent induction phase up to $100 \%$ conversion; IBVE reacted faster than pMOS (Figure 1). The polymers obtained with $\mathrm{HI} / \mathrm{I}_{2}$ initiator exhibited a very narrow, nearly monodisperse MWD with the $M_{w} / M_{n}$ ratio 1.1-1.2. Keeping their near monodispersity, they shifted progressively toward higher molecular weight with increasing monomer conversions (Figure 2A). The iodineinitiated copolymerization led to narrow but broader MWDs than those for $\mathrm{HI} / \mathrm{I}_{2}$ (Figure

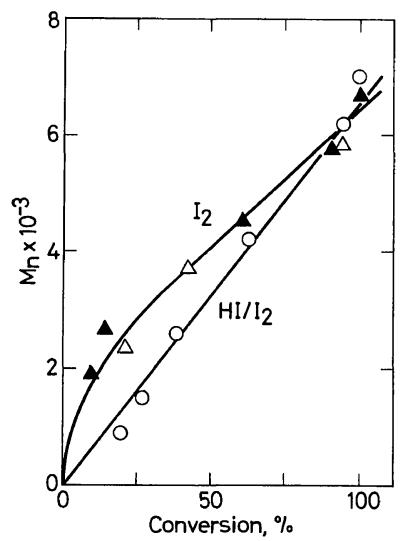

Figure 3. $M_{n}$ of IBVE-pMOS copolymers obtained at $-15^{\circ} \mathrm{C}$ as a function of total monomer conversion: $[\text { IBVE }]_{0}=0.19 \mathrm{M} ; \quad[\mathrm{pMOS}]_{0}=0.57 \mathrm{M} \quad(\mathrm{IBVE} / \mathrm{pMOS}=$ 1/3). Initiator and solvent: $(\triangle) \mathrm{I}_{2}(20 \mathrm{mM}), \mathrm{CCl}_{4} ;(\boldsymbol{\Delta}) \mathrm{I}_{2}$ $(20 \mathrm{mM})$, toluene; $(\mathrm{O}) \mathrm{HI} / \mathrm{I}_{2}(10 \mathrm{mM}$ each $), \mathrm{CCl}_{4}$. Total monomer conversion $(\%)=100 \times($ molar sum of IBVE and pMOS polymerized)/(molar sum of IBVE and pMOS in feed).

2B). A progressive increase in polymer molecular weight with conversion was also observed for iodine.

Extraction of the copolymerization products with 2-propanol [a good solvent for poly(IBVE) and a nonsolvent for poly $(\mathrm{pMOS})^{11}$ ] showed them true (random) copolymers free from the respective homopolymers. Thus, following their extraction, they gave 2-propanol-soluble and insoluble fractions, both consisting of IBVE and pMOS units of different composition (by ${ }^{1} \mathrm{H}$ NMR spectroscopy). The formation of true copolymers was further supported by the narrowness and unimodality of the MWDs coupled with almost superimposable RI and UV detector traces on SEC measurements. The latter fact indicates that UV-sensitive pMOS units are distributed uniformly in the copolymer over the entire molecular weight range.

Figure 3 (open circles and triangles) plots the $M_{n}$ of the copolymers obtained with $\mathrm{HI} / \mathrm{I}_{2}$ and iodine in carbon tetrachloride, as a function of the total monomer conversion $[100 \times$ (molar sum of IBVE and pMOS polymerized)/ 
(molar sum of IBVE and pMOS in feed)]. The $M_{n}$ was calculated from the MWD curve by assuming the uniform distribution of the two monomer units in the copolymer (see above).

The $\mathrm{HI} / \mathrm{I}_{2}$ initiating system led to a linear $M_{n}$-conversion plot passing through the origin, that is, the $M_{n}$ was directly proportional to monomer conversion, which is a typical characteristic of "living" processes. The $M_{n}$ of the iodine-initiated copolymers also increased progressively with conversion but the plot showed some curvature. These results show the $\mathrm{HI} / \mathrm{I}_{2}$ initiator to produce "living" copolymers of IBVE and pMOS with a nearly monodisperse MWD in carbon tetrachloride, whereas iodine forms long-lived polymers neither monodisperse nor perfectly living. The living IBVEpMOS copolymers thus obtained are most likely "random" in nature.

\section{Copolymerization in Toluene}

The effects of solvents and initiators on living IBVE-pMOS copolymerization were further studied in toluene, another nonpolar solvent differing from carbon tetrachloride. This aromatic solvent is better suited than carbon tetrachloride or $n$-hexane for the formation of long-lived poly(IBVE) with iodine as the initiator. ${ }^{5,12,13}$

Figure 4 compares the MWD of the IBVEpMOS copolymers formed by $\mathrm{HI} / \mathrm{I}_{2}$, iodine, and $\mathrm{BF}_{3} \mathrm{OEt}_{2}$ in toluene at $-15^{\circ} \mathrm{C}$ (total monomer conversion ca. $100 \%$; IBVE/ $p M O S=1 / 3$ in feed). The $\mathrm{HI} / \mathrm{I}_{2}$ initiator led to nearly monodisperse living copolymers whose $M_{n}$ 's were proportional to monomer conversion and nearly the same as those for carbon tetrachloride solvent. As expected, the copolymers produced by iodine showed an MWD narrower than that with carbon tetrachloride, but still broader than a monodisperse distribution. The $M_{n}$ of these polymers (Figure 3, filled triangles) increased with conversion nearly in the same way as in carbon tetrachloride.

In contrast, $\mathrm{BF}_{3} \mathrm{OEt}_{2}$ resulted in copolymers having a broad MWD with $M_{w} / M_{n}$ ratio

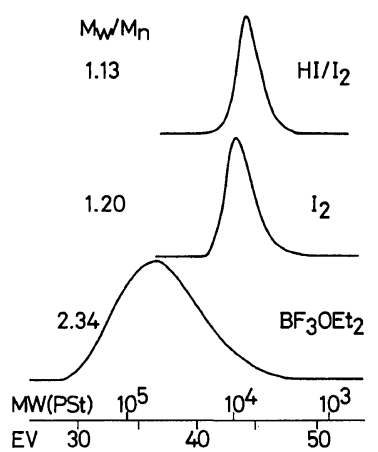

Figure 4. MWD of IBVE-pMOS copolymers obtained at $-15^{\circ} \mathrm{C}$ in toluene: $[\mathrm{IBVE}]_{0}=0.19 \mathrm{M}$; $[\mathrm{pMOS}]_{0}=$ $0.57 \mathrm{M}(\mathrm{IBVE} / \mathrm{pMOS}=1 / 3)$; total monomer conversion $=100 \%$. Initiator concentration: $\mathrm{HI} / \mathrm{I}_{2}, 10 \mathrm{mM}$ each; $\mathrm{I}_{2}, 20 \mathrm{mM} ; \mathrm{BF}_{3} \mathrm{OEt}_{2}, 1.0 \mathrm{mM} . M_{w} / M_{n}$ as indicated.

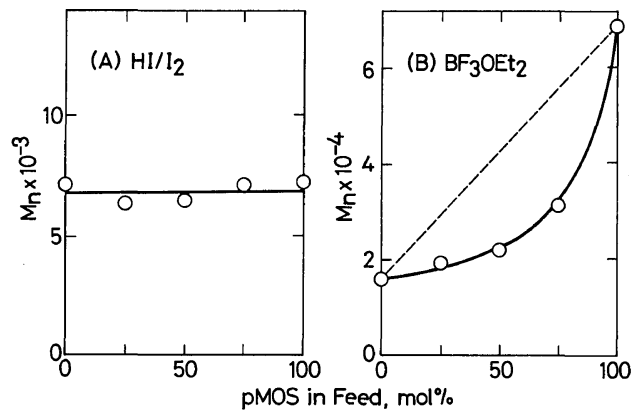

Figure 5. $M_{n}$ of IBVE-pMOS copolymers obtained at $-15^{\circ} \mathrm{C}$ as a function of IBVE/pMOS feed ratio $\left([\mathrm{IBVE}]_{0}+[\mathrm{pMOS}]_{0}=0.76 \mathrm{M}\right)$. Initiator and solvent: $(\mathrm{A})$ $\mathrm{HI} / \mathrm{I}_{2}$ (10 mM each), $\mathrm{CCl}_{4}$; (B) $\mathrm{BF}_{3} \mathrm{OEt}_{2}(1.0 \mathrm{mM})$, toluene. Total monomer conversion $=100 \%$.

around 2, and their molecular weight remained almost unchanged with increasing conversion. Thus, the metal halide initiates transfer dominant, non-living copolymerization.

\section{Effects of IBVE/pMOS Feed Ratio on Polymer Molecular Weight}

The difference between the living $\left(\mathrm{HI} / \mathrm{I}_{2}-\right.$ $\mathrm{CCl}_{4}$ or toluene) and non-living $\left(\mathrm{BF}_{3} \mathrm{OEt}_{2}-\right.$ toluene) copolymerizations became even clearer by the effects of the IBVE/pMOS feed ratio on the $M_{n}$ of the product copolymers (Figure 5). In the non-living copolymerization by $\mathrm{BF}_{3} \mathrm{OEt}_{2}$, the $M_{n}$ of the copolymers was much less than the arithmetic mean (Figure 5B, 
broken line) of the $M_{n}$ 's of the respective homopolymers formed under the same conditions. In particular, a small increase in IBVE, a more nucleophilic partner, in the feed sharply depressed the copolymer molecular weight. For example, the $M_{n}$ of the product at the IBVE/pMOS feed ratio of $1 / 3(75 \mathrm{~mol} \%$ pMOS) was less than one half that of homopoly(pMOS). Such a molecular weight depression in the copolymers should be due to frequent cross-transfer typically occurring in conventional non-living cationic copolymerization. ${ }^{1-4}$

In contrast, the living copolymers obtained with $\mathrm{HI} / \mathrm{I}_{2}$ had $M_{n}$ values almost independent of the IBVE/pMOS feed ratio and very similar to those of the respective homopolymers (Figure 5A) ${ }^{14}$ This also shows the absence of (cross-)transfer and other chain-breaking reactions in the $\mathrm{HI} / \mathrm{I}_{2}$-initiated copolymerization.

To summarize, this study has shown the $\mathrm{HI} / \mathrm{I}_{2}$ initiating system to induce not only living "homopolymerization" of vinyl and propenyl ethers ${ }^{5-8}$ but living "random copolymerization" between IBVE and pMOS to yield monodisperse copolymers.

\section{REFERENCES AND NOTES}

1. (a) T. Higashimura, "Cationic Polymerization," Kagaku Dojin, Kyoto, 1971, p 209.

(b) J. P. Kennedy and E. Maréchal, "Carbocationic Polymerization," Wiley-Interscience, New York, N.
Y., 1982, p 381.

2. (a) S. Okamura, T. Higashimura, and K. Takeda, Kobunshi Kagaku, 18, 389 (1961).

(b) S. Okamura, T. Higashimura, Y. Imanishi, R. Yamamoto, and K. Kimura, J. Polym. Sci., C, 16, 2365 (1967).

3. J. P. Kennedy and T. Chou, Adv. Polym. Sci., 21, 1 (1976).

4. T. Masuda, T. Higashimura, and S. Okamura, Polym. J., 1, 19 (1970).

5. M. Miyamoto, M. Sawamoto, and T. Higashimura, Macromolecules, 17, 265 (1984).

6. M. Miyamoto, M. Sawamoto, and T. Higashimura, Macromolecules, 17, 2228 (1984).

7. T. Higashimura, Y.-M. Law, and M. Sawamoto, Polym. J., 16, 401 (1984).

8. T. Higashimura, A. Tanizaki, and M. Sawamoto, $J$. Polym. Sci., Polym. Chem. Ed., 22, 3173 (1984).

9. (a) C. S. Marvel and G. L. Schertz, J. Am. Chem. Soc., 65, 2056 (1943).

(b) R. F. Nystrom and W. G. Brown, J. Am. Chem. Soc., 69, 1197 (1947).

10. T. Higashimura and O. Kishiro, Polym. J., 9, 87 (1977).

11. T. Higashimura, M. Mitsuhashi, and M. Sawamoto, Macromolecules, 12, 178 (1979).

12. T. Ohtori, Y. Hirokawa, and T. Higashimura, Polym. J., 11, 471 (1979).

13. T. Higashimura, M. Miyamoto, and M. Sawamoto, Polym. Prepr., Jpn., 31, 200 (1982).

14. Since the formula weight of the pMOS repeat unit is greater than that of the IBVE repeat unit (134 vs. 100), the $M_{n}$ of the pMOS-IBVE copolymers should increase with increasing pMOS content in the feed, if no chain-breaking reactions are involved in the copolymerization. The discrepancy between this expectation and the independence of the observed $M_{n}$ values on the pMOS/IBVE feed ratio is probably due to that they were determined by SEC on the basis of a polystyrene calibration. 